\title{
Continuous wave tunable fiber optical parametric oscillator with double-pass pump configuration
}

\begin{abstract}
We demonstrate a continuous wave tunable fiber optical parametric oscillator in a FabryPerot cavity consisting of a 500-m highly nonlinear fiber. In this work, the pump propagates in both directions together with the signal, thus making full use of its parametric gain. The resultant laser peak power is uneven across the wavelength range of interest due to wavelength-dependent phase modulation by the single-mode fiber sections in the cavity. This can be solved by filtering the idler spectral component from the oscillating cavity.
\end{abstract}

Keyword: Parametric oscillator, idler removal filter 